[4] E. L. Green and P. G. Cable, "Passive demodulation of optical interferometric sensors," IEEE J. Quantum Electron., this issue, pp. 1639-1644.

[5] M. Abramowitz and I. A. Stegum, Handbook of Mathematical Functions. Washington, DC: Nat. Bur. Standards.

[6] A. Dandridge, A. B. Tveten, R. O. Miles, D. A. Jackson, and T. G. Giallorenzi, "Single-mode diode laser phase noise," Appl. Phys. Lett., vol. 37, pp. 77-78, 1981.

[7] A. Dandridge and L. Goldberg, "Current induced frequency modulation in diode lasers," Electron. Lett., vol. 18, pp. 302-303, 1982.

[8] A. Dandridge, A. B. Tveten, R. O. Miles, and T. G. Giallorenzi, "Laser noise in fiber-optic interferometer systems," Appl. Phys. Lett., vol. 37, pp. 526-527, 1980.

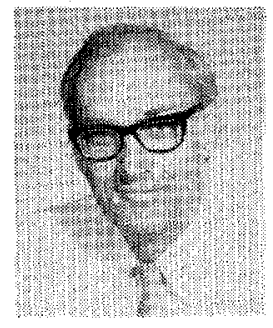

Alan B. Treten was born in Wolf Point, MT, on January 24,1934 . He received the B.A. degree from Concordia College, Moorhead, MN, the M.A. degree from the University of Nebraska, Lincoln, and the Ph.D. degree from Colorado State University, Fort Collins.

Before joining the Naval Research Laboratory, Washington, DC, in 1979, he worked as a Physics Teacher at Dana College, Mankato State University, Mankato, $\mathrm{MN}$, and Colorado State University. He has done research in secondary electron emission in metals, magnetic susceptibility of rare earth oxides, and remote sensing using light scattering from aerosols. Since 1979 he has been working with the fiber optic sensor and the optical information processing groups at NRL.
Anthony Dandridge, for a photograph and biography, see p. 564 of the April 1982 issue of this Journal.
Thomas G. Giallorenzi (SM'78), for a photograph and biography, see p. 458 of the April 1982 issue of this JouRnal.

\title{
Recent Developments in Monolithic Integration of InGaAsP/InP Optoelectronic Devices
}

\author{
UZIEL KOREN, SHLOMO MARGALIT, T. R. CHEN, K. L. YU, AMNON YARIV, FELLOW, IEEE, \\ NADAV BAR-CHAIM, KAM Y. LAU, MEMBER, IEEE, AND ISRAEL URY
}

(Invited Paper)

\begin{abstract}
Monolithically integrated optoelectronic circuits combine optical devices such as light sources (injection lasers and light emitting diodes) and optical detectors with solid-state semiconductor devices such as field effect transistors, bipolar transistors, and others on a single semiconductor crystal. Here we review some of the integrated circuits that have been realized and discuss the laser structures suited for integration with emphasis on the InGaAsP/InP material system. Some results of high frequency modulation and performance of integrated devices are discussed.
\end{abstract}

\section{INTRODUCTION}

$\mathrm{T}$ $\mathrm{HE}$ monolithic integration of optoelectronic devices composed of the III-V compounds GaAlAs/GaAs and InGaAsP/InP has been a topic of considerable interest in recent years as materials and device technology has advanced. In particular, the quaternary InP/InGaAsP material system has

Manuscript received April 1, 1982; revised June 4, 1982. This work was supported in part by the Office of Naval Research and the National Science Foundation (Optical Communication Program).

U. Koren, T. R. Chen, K. L. Yu, and A. Yariv are with the California Institute of Technology, Pasadena, CA 91125.

S. Margalit was on leave at the California Institute of Technology, Pasadena, CA 91125 . He is with the Department of Electrical Engineering, Technion-Israel Institute of Technology, Haifa, Israel.

N. Bar-Chaim, K. Y. Lau, and I. Ury are with Ortel Corporation, Alhambra, CA 91803. been a subject of intense study since devices made of these materials operate in the 1.1-1.6 $\mu \mathrm{m}$ spectral region, which coincides with the optimal region for fiber optics communication systems. As progress is being made in fiber optic technology and low loss single-mode fibers become available, there is also a strong interest in developing semiconductor devices capable of launching, receiving, and processing optical signals at the extremely high data rates that are possible with fiber optic transmission systems. The monolithic integration of optoelectronic devices on a single semiconductor crystal [1], [2] offers the potential advantages of improved reliability, low cost, and small size and also makes possible very fast operation by reducing the circuit parasitic reactances.

The most promising light source for optoelectronic devices is the semiconductor laser diode. This is due to the relatively high power that lasers can launch into fibers, and also because lasers can be modulated at very high frequencies. The lasers that have been studied for integration purposes are sometimes fabricated on semi-insulating (SI) substrates such as $\mathrm{Fe}$ doped InP or Cr doped GaAs. These crystals have very high specific resistivities which can be of the order of $10^{8} \Omega \cdot \mathrm{cm}$ for $\mathrm{Fe}$ doped InP. Although several optoelectronic devices have been integrated on conductive substrates (some will be mentioned in Sections III and IV), the SI substrates offer some important advantages. The advantages of the use of SI sub- 
strates for integration are that different devices can be electrically isolated easily, and that parasitic capacitances are largely eliminated. This point will be discussed further in the present paper.

For lasers to be considered as candidates for integrated optoelectronic circuits, they must fulfill several requirements. It is important that these lasers have low threshold currents and be capable of stable single-mode operation. The low threshold requirement results from the necessity to mount the integrated optoelectronic chip on its heat sink with the circuit side up so that wire bonding may be performed to the circuit. Heat sinking is less effective in this configuration than in the case of conventional conductive substrate lasers which are usually bonded with the laser side in direct contact with the heat sink. Another consideration is that the laser modulation speed depends on the photon stimulated lifetime in the laser cavity which is directly related to the ratio $I / I_{\text {th }}$. Consequently, lasers capable of operating at large values of $I / I_{\text {th }}$ (high power operation) are desirable.

In the second section of this paper several structures of injection laser diodes in SI substrates will be described and the operational results will be discussed briefly. In Sections III and IV we review some of the integrated structures that have been realized which include such devices as optical sources and detectors, field effect transistors (FET's), and bipolar transistors. In the last section we discuss briefly some aspects of high frequency modulation and performance of integrated devices.

\section{Lasers on Semi-Insulating Substrates}

The use of SI substrates for monolithic integration of optoelectronic devices is desirable because it facilitates the electrical isolation of the integrated components and because it is compatible with FET technology. For high frequency applications, the use of a SI substrate is important because the parasitic capacitance of interconnections can be minimized. The first demonstration of an injection laser on SI GaAs [3] was done using the crowding effect confinement structure. Since then, several other laser structures on SI substrates have been reported, including the buried heterostructure $(\mathrm{BH})$, the transverse junction stripe (TJS) structure, and groove type laser structure.

The $\mathrm{BH}$ laser on conductive $\mathrm{GaAs}$ and InP substrates has been studied intensively since it can achieve very low threshold currents and stable transverse mode operation. These excellent properties have also been obtained with SI substrates of GaAs [4] and InP [5]. The structure of a $\mathrm{BH}$ laser on SI InP reported by Matsuoka et al. [5] is shown in Fig. 1. This structure is obtained with two liquid phase epitaxial (LPE) growths. The process is similar to the fabrication of $\mathrm{BH}$ lasers on conductive n-type substrates except for the method of forming the n-type electrode. Here the first growth is of a thick $(7 \mu \mathrm{m})$ n-type InP confining layer, an InGaAsP active layer, a p-type InP confining layer, and an InGaAsP cap layer. Then, the expitaxial layers are mesa-etched down to the first $\mathrm{n}$-type InP layer and the sidewalls of the mesa are buried by the second LPE growth. About $5 \mu \mathrm{m}$ of the first n-type layer are left after etching to serve as the n-type electrode. For the

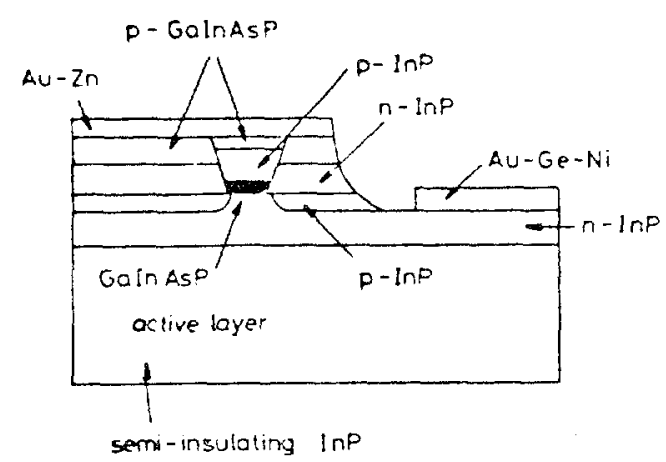

Fig. 1. Schematic structure of a BH laser on SI InP.

reduction of ohmic resistance, the distance between the laser and the etched step should be minimized (it is $\sim 25 \mu \mathrm{m}$ in this work) and the first n-type layer should be as thick as possible. This results in a series resistance of 5-6 $\Omega$ for these lasers. The minimum threshold current obtained under $\mathrm{CW}$ operation is $38 \mathrm{~mA}$ with $200 \mu \mathrm{m}$ cavity length and $2.5 \mu \mathrm{m}$ cavity width. The operating wavelength of the lasers is $1.5 \mu \mathrm{m}$.

Another laser structure that has been realized on SI GaAs substrates is the TJS structure [6], [7]. In the InP/InGaAsP system, however, the few TJS lasers that have been reported to date [8], [9] possess high threshold currents, the lowest being $250 \mathrm{~mA} \mathrm{[9].} \mathrm{This} \mathrm{is} \mathrm{due} \mathrm{to} \mathrm{leakage} \mathrm{through} \mathrm{the} \mathrm{homo-}$ junctions in the InP cladding layers [8].

A new type of laser structure recently reported on SI $\operatorname{In} P$ substrates is the groove and the groove TJS laser structure $[10]-[12]$. These lasers share some properties in common with both the $\mathrm{BH}$ and TJS configurations. The groove lasers depend on confinement by a real index profile buried optical waveguide and an effective carrier confinement by the surrounding SI material. However, like the TJS structure, they also contain a lateral $\mathrm{Zn}$ diffusion which is used for the $\mathrm{p}$ contact and can provide, in some configurations, a transverse $\mathrm{p}-\mathrm{n}$ junction.

The structure reported in [10] is shown in Fig. 2. It is obtained with a single step LPE growth process using a channeled SI InP substrate. Dovetail-shaped grooves are fabricated in the [011] direction by etching through stripe openings in a $\mathrm{Si}_{3} \mathrm{~N}_{4}$ layer which is deposited on the substrate. Following etching, the $\mathrm{Si}_{3} \mathrm{~N}_{4}$ is removed from one side of the groove leaving a $\mathrm{Si}_{3} \mathrm{~N}_{4}$ mask which prevents epitaxial growth on the other side of the groove. An SEM photomicrograph showing the LPE grown layers is shown in Fig. 3. The three grown layers: p-InP, InGaAsP (undoped), and $n-\operatorname{In} \mathbf{P}$ grow inside the groove and also outside on the exposed side of the groove. The quaternary layers inside and outside the groove are disconnected, thus forming the buried crescent-shaped optical waveguide. A lateral $\mathrm{Zn}$ diffusion is performed following the growth through etched window openings as shown in Fig. 2. The diffusion front penetrates into the grown p-layer inside the groove and creates a continuous lateral path for the current to flow between the positive and negative broad contacts located on either side of the groove. With this structure current thresholds of $28 \mathrm{~mA}$ for $300 \mu \mathrm{m}$ cavity length and 3-4 $\mu \mathrm{m}$ active layer width have been obtained.

Another configuration similar to the groove laser is the 


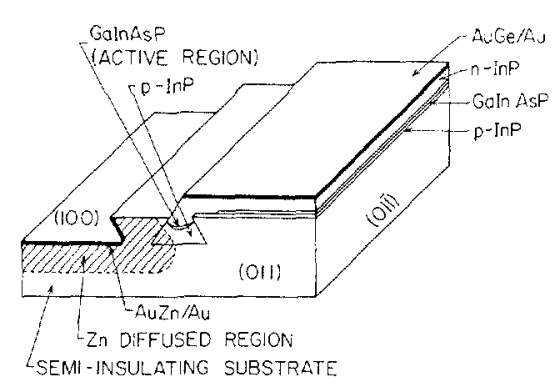

Fig. 2. Schematic structure of a groove laser on SI InP substrate.

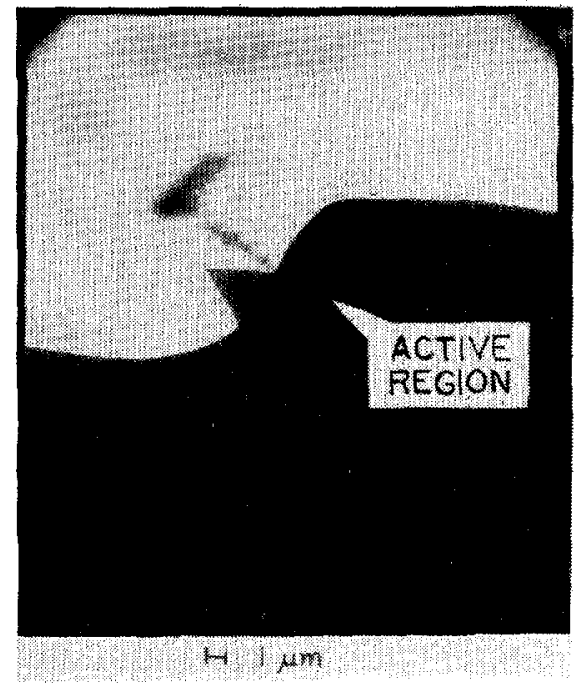

Fig. 3. SEM photomicrograph of a groove laser showing the crescentshaped active region.

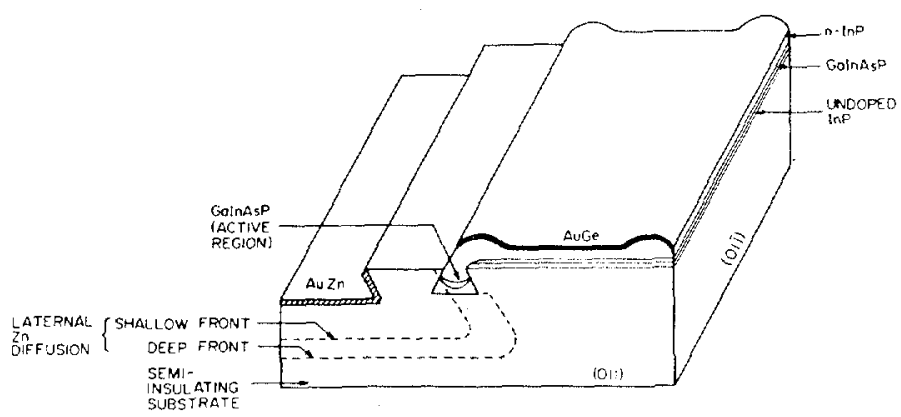

Fig. 4. A schematic description of a groove TIS laser on SI InP.

groove TJS laser shown in Fig. 4. Although the processing steps are the same as those for the standard groove laser, the difference is that here only $n$-type layers are grown and the $p-n$ junction is the transverse junction at the diffusion front.

One conclusion from several studies that have been made is that the exact location and shape of the junction, and thus of the injected carrier distribution, has certain implications concerning the thresholds and transverse modal behavior of these lasers. Two related studies have been reported. One study investigated the conditions required to achieve very low threshold lasers [11], and the other investigated the conditions required to achieve high power lasers with stable fundamental mode operation. For low threshold lasers, a very small and narrow active region is desirable. Also, the diffusion front should be located near the edge of the active region (see
Fig. 4) to minimize leakage from the top p- ${ }^{+}$InP homojunction. These lasers exhibit current thresholds of $14 \mathrm{~mA}$ for $300 \mu \mathrm{m}$ cavity length and active region dimensions of about $2 \times 0.2 \mu \mathrm{m}$. When the active region width is $2 \mu \mathrm{m}$ wide or less, fundamental transverse mode operation is observed.

The transverse junction mode of injection is found to be suitable for high power stable fundamental mode lasers [12]. High output power capability is obtained by increasing the active region dimensions to $3-5 \mu \mathrm{m}$ width and about $1 \mu \mathrm{m}$ thickness, and by locating the transverse junction near the center of the active region. A photomicrograph of this structure is shown in Fig. 5. An optical waveguide of these dimensions can support many transverse modes, but fundamental mode operation can still be maintained due to gain stabilization mechanism unique to transverse injection. Fig. 6 shows the calculated [12] distribution of carriers injected from a transverse junction located at the middle of the active region. Such a distribution prefers the fundamental mode since its overlap with the optical intensity profile is higher for the fundamental mode than for the higher order modes which carry a larger fraction of their power near the sides of the waveguide. This increases the fundamental mode gain relative to that of the higher order modes. This effect is enhanced at high optical powers since the shortening of the carrier's lifetime by stimulated emission causes the carrier distribution to become more localized at the center of the waveguide (see Fig. 6). These lasers have current thresholds of about $50 \mathrm{~mA}$ with $300 \mu \mathrm{m}$ cavity length and power outputs of $250 \mathrm{~mW} /$ facet in pulsed operation, and with stable fundamental transverse mode at these power levels. The far-field measurements at high optical powers are shown in Fig. 7.

Another method of stabilizing the transverse mode operation of lasers with lateral diffusion and buried optical waveguides is to weaken the optical guiding. This has been done with the terrace substrate InGaAsP/InP laser on SI InP [13], [14]. The laser structure shown in Fig. 8 provides weaker optical waveguiding laterally than the groove laser (Fig. 2). The configuration of [14] results in lasers with 35-50 mA threshold currents and single-mode operation at relatively high current levels are obtained. Far-field patterns of this laser are shown in Fig. 9.

\section{Integration of Optical Devices and Field}

\section{Effect Transistors on Semi-Insulating Substrates}

One common property of most of the published reports of the integration of optical devices and FET's on InP is the use of SI InP substrates, the reason being that the n-channel of the FET is very conveniently defined with an $\mathrm{n}$-type layer located directly above the SI material. Conductive substrates are more difficult to use because it has not yet been proven possible to grow high resistivity epitaxial layers on InP substrates. In the GaAs/GaAlAs material system the work of Fukuzawa et al. [15] demonstrated the integration of lasers and metal semiconductor field effect transistors (MESFET's) on conductive n-type substrates utilizing a high resistivity (200 $\Omega \cdot \mathrm{cm}) \mathrm{GaAlAs}$ insulation layer between the layers of the lasers and MESFET's. This configuration resulted, however, 




Fig. 5. SEM photomicrograph of a high power groove TJS laser.



Fig. 6. Calculated carrier distribution for a transverse $p-n$ junction located at the middle of the waveguide. The assumed (parabolic) optical profile is shown above.

in several nonnegligible parasitic impedances and leakage currents [15] which can be minimized by the use of SI substrates.

Several reports have been published recently [16]-[18] on the integration of optical detectors and FET's on SI substrates. Leheny et al. [16] describe a p-i-n photodiode integrated with a junction field effect transistor (JFET) using an LPE grown InGaAs layer on SI InP. A shallow $\mathrm{Zn}$ diffusion is used to fabricate the $\mathrm{p}-\mathrm{i}-\mathrm{n}$ junction and the $\mathrm{Zn}$ diffused part is extended to create the gate of the JFET. This device is shown schematically in Fig. 10. Transconductance of $50 \mathrm{~m} \mho / \mathrm{mm}$

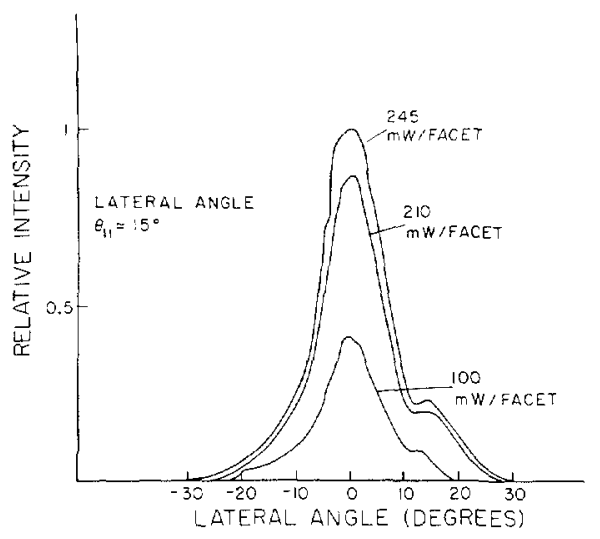

Fig. 7. Far-field intensity distributions at high powers for a groove TJS laser.

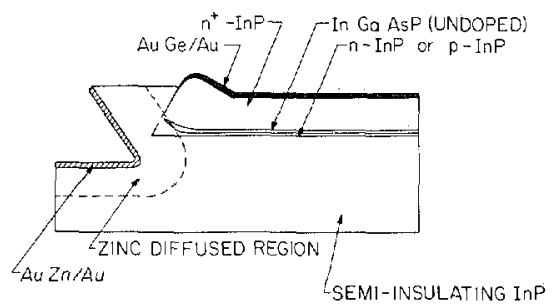

Fig. 8. Schematic structure of a terrace substrate laser on SI InP.

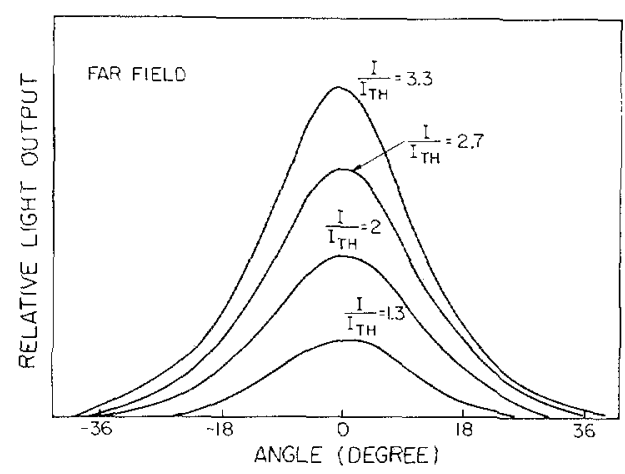

Fig. 9. Far-field intensity distribution for the terrace substrate laser.

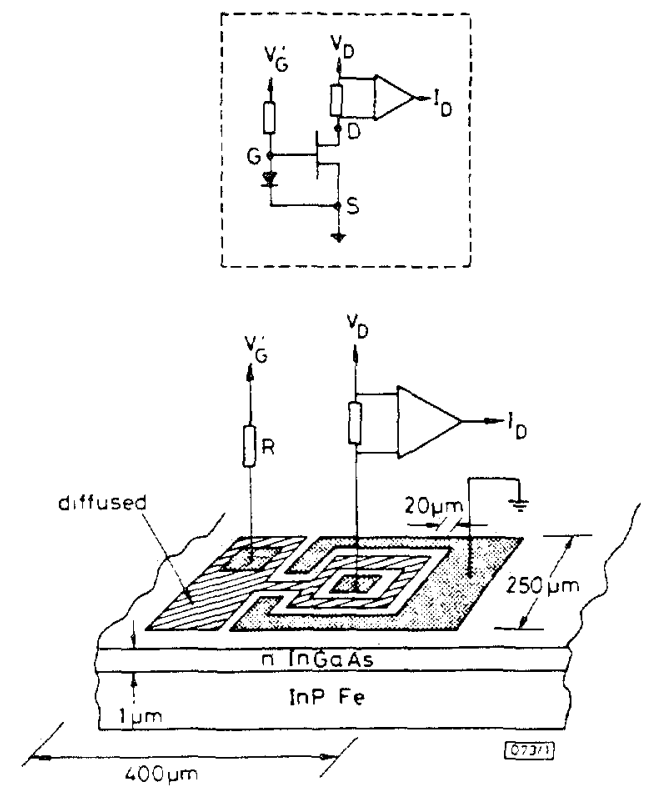

Fig. 10. Schematic structure of an integrated $\mathrm{p}-\mathrm{i}-\mathrm{n}$ FET optical receiver on SI InP substrate. 
gate width with $7 \mu \mathrm{m}$ gate length are obtained. The p-i-n diodes exhibit a dark current of less than $100 \mathrm{nA}$ at $10 \mathrm{~V}$ reverse bias voltage.

A different approach reported by Barnard et al. [17] demonstrates the integration of a photoconductor optical detector with a dual gate MESFET. Layers of InAlAs and InGaAs are grown by molecular beam epitaxy in SI InP. The InGaAs layer is used to form the photoconductive material and also the n-channel of the FET. The MESFET is used here as a voltage preamplifier with the additional gate reserved for other applications such as automatic gain control for monitoring a source or for multiplexing purposes in which several of these devices with common drains can drive a single laser diode.

An integration of a groove TJS laser diode with a metal insulator semiconductor field effect transistor (MISFET) on SI InP has been recently reported [19]. The structure is shown schematically in Fig. 11. The n-channel of the MISFET is obtained by etching a recess through the top n-InP and quaternary layers and into the undoped n-type InP layer. The recess length is $6 \mu \mathrm{m}$ and the gate length is $1.5 \mu \mathrm{m}$. Typical transconductance of $10 \mathrm{~m} \mho$ with $200 \mu \mathrm{m}$ channel width are obtained. The groove lasers with $15 \mathrm{~mA}$ threshold currents have been modulated at relatively low frequencies by the MISFET's at twice the threshold current with no additional biasing current, as shown in Fig. 12.

In work reported recently by Carter et al. [18], several optoelectronic devices such as LED's, detectors, resistors, and MESFET's have been integrated monolithically on a SI GaAs wafer. The FET layers are grown by vapor phase epitaxy, followed by LPE growth for the heterojunction diodes. Using these devices as building blocks, several optoelectronic integrated circuits such as LED-transmitter, and p-i-n FET receiver and repeater have been demonstrated.

\section{Integration of Optical and Bipolar Devices}

Heterojunction bipolar transistors possess the advantages of high emitter injection efficiency and compatibility with the double heterostructure of the laser diode. Since the emitter is made of the high bandgap material, the injection efficiency is practically independent of the emitter and base doping and thus, the base doping can be increased [20]. Increasing the base doping level results in minimized base spreading resistance, while a decrease of the emitter doping reduces the junction capacitance. Both effects improve the frequency response of the device. Bipolar heterojunction transistors can also be used as high gain photodetectors. The incoming light is absorbed in the low bandgap base and in the depleted part of the collector. Several structures of heterojunction phototransistors (HPT's) in InGaAsP have been reported [21]-[24]. The structure of the device reported by Sasaki et al. [22] is shown in Fig. 13,

A natural way to integrate a bipolar transistor with a $\mathrm{BH}$ laser is to use the regrowth process of the laser to form the HPT. Such a structure has been reported recently in the GaAs/GaAlAs system [25]. The structure is shown schematically in Fig. 14. The layers that are regrown for the reverse biased junctions of the $\mathrm{BH}$ laser are also used for the heterojunction transistor. These layers are n-GaAs (collector), p-GaAs (base), and n-GaAlAs (emitter). The base contact is

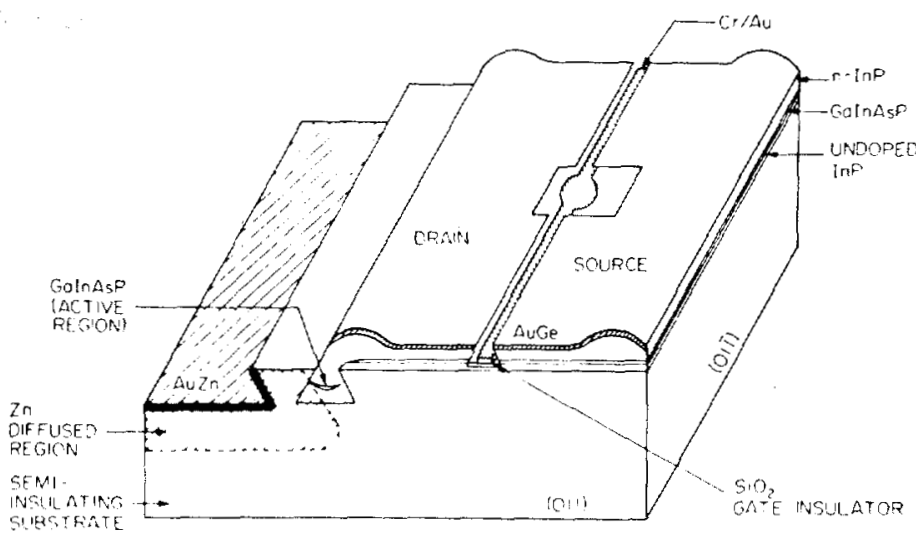

Fig. 11. Schematic structure of an integrated groove laser and MISFET on SI InP substrate.

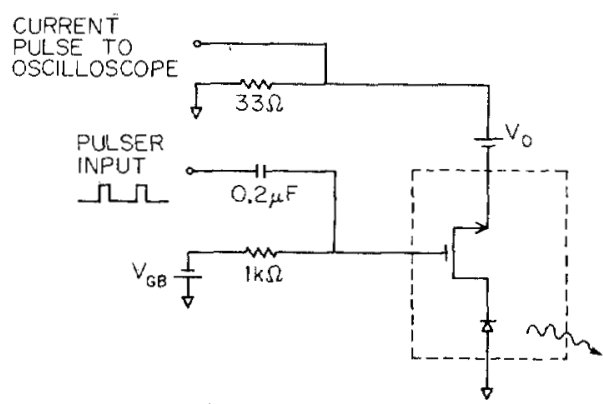

(a)

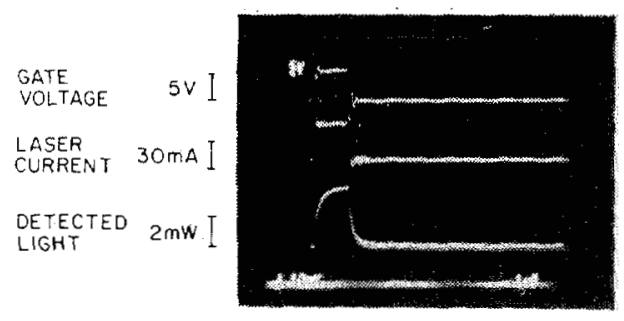

(b)

Fig. 12. Modulation of the laser by the FET. (a) The modulation circuit. (b) Modulation response, horizontal scale $-1 \mu \mathrm{s} /$ div. The response time is limited by the photodiode.

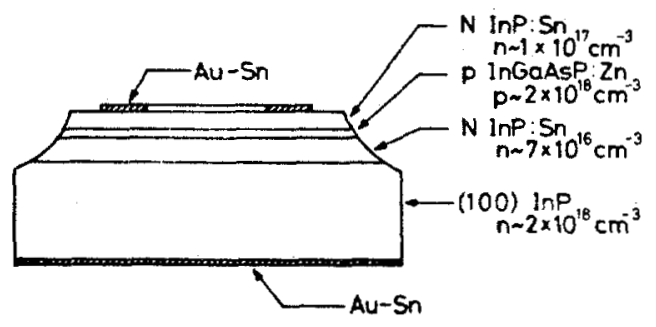

Fig. 13. Schematic structure of an InGaAsP/InP HPT.

achieved by a $\mathrm{Zn}$ diffused area that is made to reach the p-GaAs base layer. The transistors can be used as drivers for the laser diodes and also as photoreceivers with part of the emitter electrode removed so that the base and collector layers can absorb the incoming light. The incoming light is thus amplified by the integrated device. With this structure current gains of the order of $100-400$ are obtained with collector current levels of a few $\mathrm{mA}$.

The response time of the phototransistor is determined by the lifetime of excess minority carriers in the base region and it is of the order of a few ns [21]. This is relatively slower 


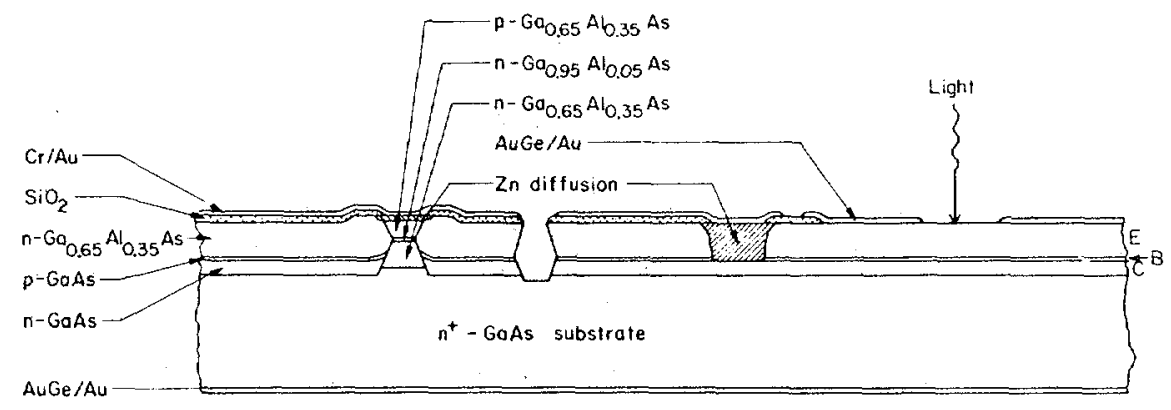

Fig. 14. Schematic structure of a $B H$ laser integrated with a bipolar transistor on conductive GaAs substrate.

than the expected response of a p-i-n FET or an avalanche photodiode-FET configuration. However, it has been argued [23] that the phototransistor can achieve as high a signal-tonoise ratio as that which is obtainable with p-i-n FET's and APD-FET's at data rates up to $700 \mathrm{Mbits} / \mathrm{s}$.

Another approach which has been suggested by Sasaki et al. [22] is to grow the layers of the phototransistor directly above the layers of the light source (DH laser or LED) on conductive InP substrate so that the vertical current flow in the phototransistor will also drive the light source. This structure is suggested for applications such as light amplification and frequency conversion.

\section{INTEGRATED MIRRORS}

The cavity of a semiconductor injection laser is normally obtained by cleaving a semiconductor wafer along one of the crystal cleavage planes. The two parallel planes confining the wafer provide the optical mirrors of the laser. This technique, although very useful, is not ideally suited for complex integrated circuits since it imposes severe limitations on the size and geometry of the semiconductor wafer. The fabrication of the mirrors by alternative processes removes these limitations and permits the integration of the laser cavity with much more flexibility in a complex integrated device. Injection lasers with chemically etched mirrors have been reported in the InGaAsP/InP system. A recent paper describing these works is given by Wright et al. [26]. The mirrors' fabrication processes include both wet chemical techniques and the socalled dry processes such as plasma, reactive ion, and sputter etching techniques. Using a wet chemical etching technique, Wright et al. [26] demonstrated the integration of lasers and photodiodes (using the same double heterostructure for both devices) on a single n-type $\ln P$ substrate. In this configuration, the photodiodes can be used to monitor the laser light output. These devices have also been operated as a combined laser oscillator and resonant laser amplifier. CW operation of InGaAsP/InP etched mirror lasers has been demonstrated by Iga et al. [27]. The application of this technique for obtaining short cavity lasers and for monolithically integrating lasers with monitoring photodetectors has been reported. Recently, a promising technique [28] using a combination of reactive ion etching and wet crystal stop etch planes process has been reported. This process leads to (011) etched mirrors in $\operatorname{InP} /$ InGaAsP that can provide mirrors for important laser structures (e.g., BH lasers).

Another approach reported recently for the GaAs/GaAlAs system is the microcleaved facets (MCF) process [29], [30]. This technique is based on cleaving a suspended DH cantilever at the ends of the laser structure by means of ultrasonic vibrations. The cantilevers break at the crystanl cleavage planes thus providing cleaved, high quality mirrors for the laser cavity. Preliminary results [31] in the InGaAsP/InP system have also been obtained with the MCF technique. These results were obtained with groove lasers on SI InP substrates similar to those discussed in Section II (Fig. 2), but with the wafer masked by $\mathrm{Si}_{3} \mathrm{~N}_{4}$ on both sides of the groove during epitaxy so that expitaxial growth occurs only inside the groove (Fig. 15). The microcleavage technique that was used here is somewhat different from those reported earlier [29], [30] which were based on selective etching. Here, miniature bridges are formed by etching beneath a $15 \mu \mathrm{m}$ wide $\mathrm{Si}_{3} \mathrm{~N}_{4}$ stripe which covers the laser groove. This etching is done in the original SI InP substrate and does not intersect any grown layers, so that sharp, well defined, dovetail-shaped directional etching is obtained on both sides of the $\mathrm{Si}_{3} \mathrm{~N}_{4}$ stripe. When the etchings from both sides join, triangular sispended bridges are formed. Microcleavage is then accomplished by use of ultrasonic vibrations. The microcleaved structure is shown schematically in Fig. 15 and an SEM photomicrograph of the triangular microcleaved facet is shown in Fig. 16. Threshold currents of $70 \mathrm{~mA}$ have been obtained for lasers with two microcleaved mirrors for a cavity length of $140 \mu \mathrm{m}$ and an active region cross section of $4 \times 0.9 \mu \mathrm{m}$. Lower threshold currents can in principle be achieved by reducing the lateral dimensions of the active region.

\section{High Frequency Performance of Integrated Optoelect Ronic Circuits}

In this section, the high frequency characteristics of some basic transmitter and receiver circuits are discussed. The considerations here are sufficiently general as to be applicable to InP optoelectronic circuits as well as those in GaAs. A laser diode can be driven directly at the $\mathrm{GHz}$ frequency range by a microwave modulation signal [32], [33]. Another way to obtain high frequency modulation of the light output of InGaAsP/InP lasers has been reported by Tsang et al. [34] utilizing an intracavity electroabsorption modulator. For direct modulation the equivalent circuit of the laser diode with the packaging impedances is shown in Fig. 17. The modulated light output is proportional to the current $i_{p}$ through the inductor $L$ [35]. The ratio of $i_{p}$ to the total current $i$ through the diode is the familiar small signal modula- 


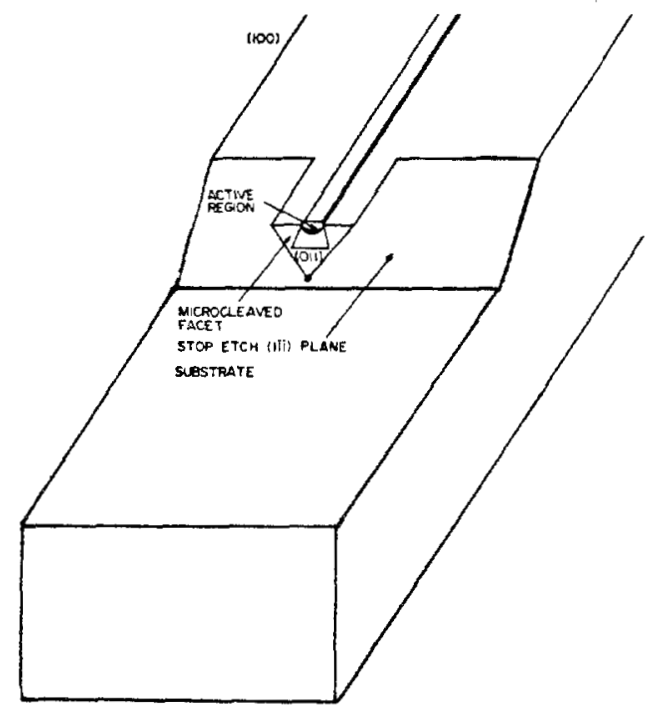

Fig. 15. Schematic structure of a groove laser with microcleaved mirrors.

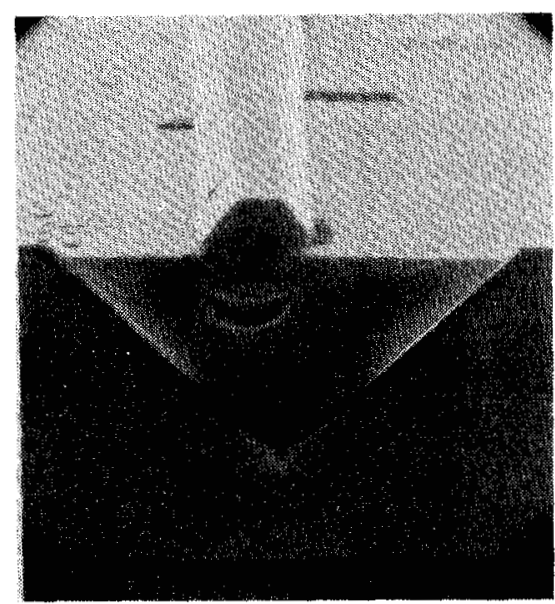

Fig. 16. SEM photomicrograph of a microcleaved mirror.

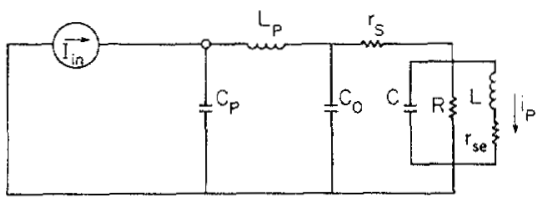

Fig. 17. Equivalent circuit of a laser diode and its packaging for high frequency modulation.

tion response of the intrinsic laser diode [36], displaying a resonance at the frequency $f=1 /(2 \pi \sqrt{L C})$. The combined impedance of the RLC circuit is small compared to the parasitics so that in analyzing the effect of parasitic elements on the modulation performance, the laser diode can be approximated as a short circuit, which reduces the equivalent circuit in Fig. 17 to that described in [32]. The circuit elements $r_{s}, C_{o}, L_{p}, C_{p}$ in Fig. 17 are the laser series resistance, laser parasitic capacitance, bond wire inductance, and laser package parasitic capacitance, respectively. These external elements form a bypassing and blocking network which isolates the laser diode from the modulation drive signal at high frequencies, and this is believed to be a cause of the observed dip [37] in the modulation response of some lasers. The reac- tances $L_{p}$ and $C_{p}$ are typically of the order of $1-2 \mathrm{nH}$ and $0.2-0.5 \mathrm{pF}$, respectively. Analysis and experiment [38] show that these package parasitic elements would not reduce the modulation bandwidth of the laser up to at least $4 \mathrm{GHz}$ (although, for direct modulation, if $L_{p}$ and $C_{p}$ are not minimized, they may present a problem since the circuit of Fig. 17 cannot then be matched to a $50 \Omega$ transmission line at this frequency range). In general, well designed microwave packages which permit high frequency operation are already standard items in microwave technology [39], so that package parasitics are not considered to be a limitation for high speed operation of optoelectronic devices. A more serious limitation is due to the parasitic laser capacitance $C_{o}$ and the series resistance $r_{s}$, both of which should be minimized for high speed operations. Values of $r_{s}$ as small as $10 \Omega$ can be routinely produced with the relatively well-developed laser contact technology. The parasitic capacitance $C_{o}$, however, depends strongly on laser structures. For example, the measured value of $C_{o}$ in common stripe geometry lasers on conductive substrates lies between 30 and $100 \mathrm{pF}$, while that of the $\mathrm{BH}$ laser is reduced to about $10 \mathrm{pF}$ [32] due to the presence of a blocking layer outside the active region. Further reduction in $C_{o}$ becomes possible for lasers in optoelectronic integrated circuits by virtue of small contact pads and their fabrication on SI substrate. Examples of such lasers have been given in Section II of this paper. A recently reported GaAs BH laser monolithically integrated with a MESFET on SI GaAs [40] possesses a parasitic capacitance $C_{O}$ as low as $4 \mathrm{pF}$. This circuit is shown in Fig. 18. The modulated laser output is detected with a fast avalanche photodiode and the detected signal is compared to the drive signal using a network analyzer. The measurement results are shown in Fig. 19, where the data have been compensated for the frequency response of a precalibrated avalanche diode. Modulation frequencies exceeding $4 \mathrm{GHz}$ with optical modulation depths beyond 70 percent have been obtained. One distinct advantage of this integrated circuit, from system considerations, is that by terminating the gate of the MESFET with $50 \Omega$, the impedance of this device can be matched to the driving source over a very wide band (from $0-4 \mathrm{GHz}$ ). The equivalent circuit with a simplified representation of the FET is shown in Fig. 20 where $g_{m}$ is the transconductance and $C_{g}$ is the effective gate capacitance. In contrast to this it is impossible to match the impedance of the solitary diode (Fig. 17) over any extended frequency range. The measured VSWR of the device in Fig. 18 is lower than 1.15 over the entire band.

In the above discussion, we have considered the effect of parasitic elements on the modulation response of lasers in optoelectronic circuits. We have not addressed fundamental limitations on the high speed operation of the laser itself. This subject is still under investigation, although preliminary results [38], [40], exemplified by the laser-MESFET circuit described above, indicate that laser diodes can be reliably modulated at a much higher rate than the commonly accepted bandwidth of $2-3 \mathrm{GHz}$. It is expected that, due to the increased reliability and high-power tolerance, InGaAsP lasers can be modulated at even higher rates than those in GaAs. Experimental efforts are underway to explore this possibility. 


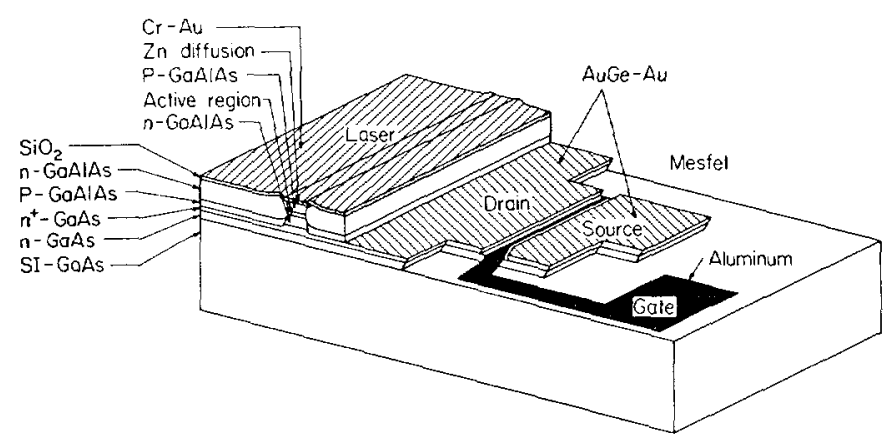

Fig. 18. Schematic structure of a BH laser integrated with a MESFET on SI GaAs.

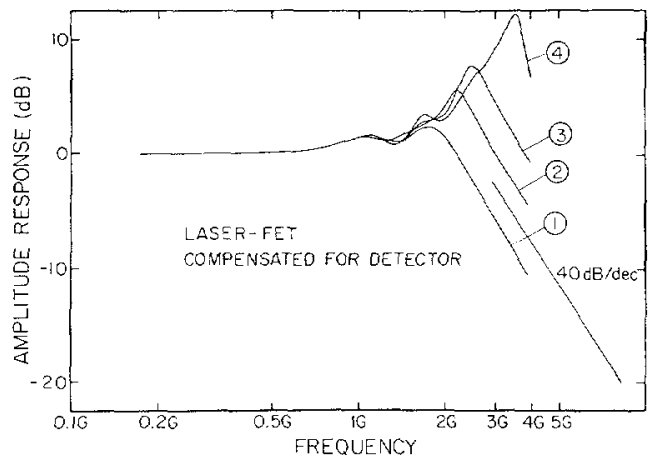

Fig. 19. High frequency response of the integrated device at different bias currents. The current values are (1) $-1.25 I_{\mathrm{th}}$, (2) $-1.35 I_{\mathrm{th}}$, (3) $-1.55 I_{\mathrm{th}}$, (4) $-1.7 I_{\mathrm{th}}$.

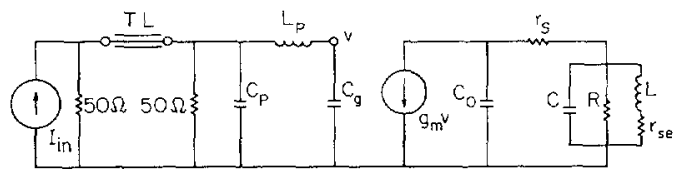

Fig. 20. Equivalent circuit of a monolithically integrated laser FET device.

For an optical receiver circuit, signal-to-noise $(S / N)$ considerations are important as well as the frequency response function. The equivalent circuit for an optical receiver similar to that given by Personick et al. [41] is shown in Fig. 21. In this equivalent circuit, $C_{O}$ is the photodiode junction capacitance and $R_{L}, C_{g}$ are the load resistance and amplifier input capacitance, respectively. The amplifier is assumed to be an infinite input impedance voltage amplifier and its noise is represented by current and voltage noise sources. The thermal noise of the load resistor is included in this current noise source. The photodiode is represented by signal and noise current sources in parallel. The amplifier for this circuit can be, in principle, an integrated FET preamplifier. One can see that the reduction of the inductance $L_{P}$ achieved by integration will result in higher bandwidth and increased $S / N$ ratio. Another way to increase the $S / N$ is to reduce the photodiode capacitance $C_{o}$ by reducing its area and its parasitic contact capacitance (using a SI substrate). This improves the $S / N$ ratio because the signal voltage will increase while the voltage noise $e_{n}$ remains fixed (see Fig. 21).

In conclusion, we have reviewed some of the recent progress being made in the area of integrated optoelectronic circuits

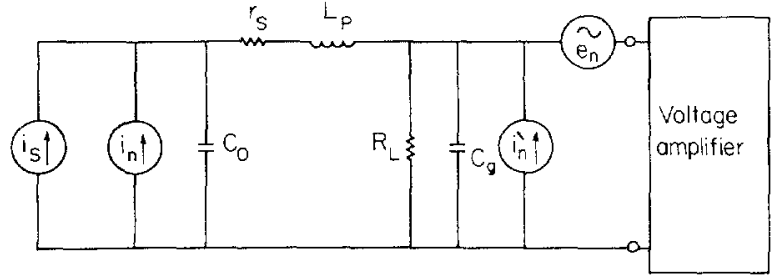

Fig. 21. Equivalent circuit for a high frequency optical receiver.

which incorporate transistors, detectors, and light emitting diodes, with emphasis on the InGaAsP/InP material system. Devices made of these materials operate in the spectral region which coincides with the optimal region for fiber optic telecommunication systems. This technology offers the potential advantages of improved performance for optoelectronic devices, providing a natural interface between electronic equipment and fiber optic systems. In the long range, this technology may also lead to the realization of complex integrated optoelectronic circuits having low cost and improved reliability. It appears that optoelectronic devices will play an important role in high-frequency high-performance fiber optic communication systems.

\section{REFERENCES}

[1] A. Yariv, "Active integrated optics," in Proceedings of 1971 Esfahan Conference on Pure and Applied Physics, M. Feld, N. Kumit, and A. Javan, Eds. Cambridge, MA: MIT Press, 1972.

[2] - "Components of integrated optics," Laser Focus, pp. 4042, Dec. 1972

[3] C. P. Lee, S. Margalit, and A. Yariv, "Double heterostructure GaAs-GaAlAs injection lasers on semi-insulating substrate using carrier crowding," Appl. Phys. Lett., vol. 31, pp. 281-282, 1977.

[4] N. Bar-Chaim, J. Katz, I. Ury, and A. Yariv, "Buried heterostructure GaAlAs lasers on semi-insulating substrates," Electron. Lett., vol. 17, pp. 108-109, 1981.

[5] T. Matsuoka, K. Takahei, Y. Noguchi, and H. Nagal, "1.5 micton region InP/GalnAsP buried heterostructure lasers on semiinsulating substrates," Electron. Lett., vol. 17, pp. 12-14, 1981.

[6] C. P. Lee, S. Margalit, I. Ury, and A. Yariv, "GaAs-GaAlAs injection lasers on semi-insulating substrate using laterally diffused junctions," Appl. Phys. Lett., vol. 32, pp. 410-412, 1978.

[7] T. Kumabe, T. Tanaka, H. Namizaki, S. Takamika, M. Ishii, and W. Susaki, "High temperature single mode cw operation of a TJS laser using a semi-insulating GaAs substrate," Japan. J. Appl. Phys., vol. 58, pp. 371-375, 1979.

[8] D. J. Bull, N. B. Patel, F. C. Prince, and Y. Nannichi, "Oxide defined TJS laser in InGaAsP/InP DH structures," IEEE $J$. Quantum Electron., vol. QE-15, pp. 710-713, 1979.

[9] S. Sakai, T. Aoki, and M. Umeno, "Dual wavelength InGaAsP/ InP TIS lasers," Electron. Lett., vol, 18, pp. 18-19, 1982.

[10] K. L. Yu, U. Koren, T. R. Chen, P. T. Chen, and A. Yariv, "Groove GaInAsP laser on semi-insulating InP," Electron. Lett., vol. 17, pp. 790-792, 1981.

[11] _- "A groove GaInAsP laser on semi-insulating InP using a laterally diffused junction," IEEE $J$. Quantum Electron., vol. QE-18, pp. 817-820, May 1982.

[12] T. R. Chen, U. Koren, K. L. Yu, K. Y. Lau, L. C. Chiu, A. Hasson, S. Margalit, and A. Yariv, "High power InGaAsP/InP groove transverse junction laser with gain stabilized single mode operation," Appl. Phys. Lett., Aug. 1982.

[13] T. Matsuoka, Y. Suzuki, Y. Noguchi, and H. Nagai, "GaInAsP/ InP DH lasers on semi-insulating InP substrate with terrace structure," Electron. Lett., vol. 18, pp. 359-361, 1982.

[14] T. R. Chen, K. L. Yu, U. Koren, A. Hasson, S. Margalit, and A. Yariv, "Mode stabilized terrace InGaAsP lasers on semi-insulating InP," Appl. Phys. lett., submitted for publication.

[15] T. Fukuzawa, M. Nakamura, M. Hirao, T. Kuroda, and J. Umeda, "Monolithic integration of a GaAlAs injection laser with a 
Schottky gate field effect transistor," Appl. Phys. Lett., vol. 36, pp. 181-183, 1980.

[16] R. F. Leheny, R. E. Nahory, M. A. Pollack, A. A. Ballman, E. D. Beebe, T. C. Dewinter, and R. J. Martin, "Integrated InGaAs p-i-n FET photoreceiver," Electron. Lett., vol. 16, pp. 353-355, 1980 .

[17] J. Barnard, H. Ohno, E. C. Wood, and L. F. Eastman, "Integrated heterostructure GaInAs photoreceiver with automatic gain control," IEEE Trans. Electron. Devices Lett., vol. EDL-2, pp. $7-9,1981$

[18] A. C. Carter, N. Forbes, and R. C. Goodfellow, "Monolithic integration of optoelectronic, electronic and passive components in GaAlAs/GaAs layers," Electron. Lett., vol. 18, pp. 72-74, 1982.

[19] U. Koren, K. L. Yu, T. R. Chen, N. Bar-Chaim, S. Margalit, and A. Yariv, "Monolithic integration of very low threshold GaInAsP laser and MISFET on semi-insulating InP," Appl. Phys. Lett., vol. 40, pp. 643-645, 1982.

[20] H. Kroemer, "Heterostructures for everything, device principle of the 1980's," Japan. J. Appl. Phys., vol. S20-1, pp. 9-13, 1981.

[21] J. C. Campbell, A. G. Dental, C. A. Burrus, and J. F. Ferguson, "InP/InGaAs heterojunction phototransistor," IEEE $J$. Quantum Electron., vol. QE-17, pp. 264-269, Feb . 1981.

[22] A. Sasaki and M. Kuzuhara, "InGaAsP/InP heterojunction phototransistors and light amplifiers," Japan. J. Appl. Phys., vol. 20, pp. L283-L286, 1981.

[23] K. Tabatabaie-Alavi and C. G. Fonstad, "Performance comparison of heterojunction phototransistors, p-i-n FET's and APDFET's for optical fiber communication systems," IEEE $J$. Quantum Electron., vol. QE-17, pp. 2259-2261, Dec. 1981.

[24] M. Tobe, A. Ameniya, S. Sakai, and M. Umens, "High sensitivity InGaAsP/InP phototransistors," Appl. Phys. Lett., vol. 37, p. 7375, 1980.

[25] N. Bar-Chaim, Ch. Harder, J. Katz, S. Margalit, I. Ury, and A. Yariv, "Monolithic integration of a GaAlAs buried heterostructure laser and a bipolar phototransistor," Appl. Phys, Lett., vol. 40 , pp. 556-558, 1982.

[26] P. D. Wright, R. J. Nelson, and R. B. Wilson, "Monolithic integration of InGaAsP heterostructure lasers and electrooptical devices," IEEE J. Quantum Electron., vol. QE-18, pp. 249258, Feb. 1982

[27] K. Iga and B. I. Miller, "Chemically etched-mirror GaInAsP/InP lasers-Review," IEEE J. Quantum Electron., vol. QE-18, pp. 22-30, Jan. 1982.

[28] L. A. Coldren, K. Furuya, B. I. Miller, J. A. Rentschler, A. H. Dayem, and P. Mankiewich, "Etched mirrors, grooves and surfaces for GaInAsP/InP integrated optical devices using stop etch crystal planes," presented at the Int. and Guided Wave Optics Conf., Pacific Grove, CA, Jan. 1982, paper WB1.

[29] H. Blauvelt, N. Bar-Chaim, D. Fekete, S. Margalit, and A. Yariv, "AlGaAs lasers with microcleaved mirrors suitable for monolithic integration," Appl. Phys. Lett., vol. 40, pp. 289-290, 1982.

[30] O. Wada, S. Yamakoshi, T. Fujii, S. Hiyamizu, and T. Sakurai, "AlGaAs/GaAs microcleaved facet (MCF) laser monolithically integrated with photodiode," Electron. Lett., vol. 18, pp. 189$190,1982$.

[31] U. Koren, A. Hasson, K. L. Yu, T. R. Chen, S. Margalit, and A. Yariv, "InGaAsP/InP microcleaved groove laser suitable for monolithic integration," submitted to the Eighth IEEE Int. Semiconductor Laser Conf., Sept. 1982.

[32] M. Maeda, K. Nagano, M. Tanaka, and K. Chiba, "Buried heterostructure laser packaging for wideband optical transmission systems," IEEE Trans. Commun., vol. COM-26, pp. 1076-1081, 1978 .

[33] K. Saito and R. Ito, "Buried heterostructure AlGaAs lasers," IEEE J. Quantum Electron., vol. QE-16, pp. 205-215, Feb. 1980.

[34] D. Z. Tsang, J. N. Walpole, S. H. Groves, J. J. Hsieh, and J. P. Donnelly, "Intracavity loss modulation of GaInAsP diode lasers," Appl. Phys. Lett., vol. 38, pp. 120-122, 1981.

[35] J. Katz, S. Margalit, C. Harder, D. Wilt, and A. Yariv, "The intrinsic electrical equivalent circuit of a laser diode," IEEE $J$. Quantum Electron., vol. QE-17, pp. 4-7, Jan. 1981.

[36] H. Kressel and J. K. Butler, Semiconductor Lasers and Heterojunction LEDs. New York: Academic, 1977, ch. 17.

[37] Hitachi, HLP Laser Diode Application Manual, p. 22.

[38] K. Y. Lau, Ch. Harder, S. Margalit, and A. Yariv, "Very high speed lasers in optoelectronic integrated circuits," to be published.

[39] R. J. Akello, B. Easter, and I. M. Stephenson, "Experimental measurement of microstrip transistor-package parasitic reactances," IEEE Trans. Microwave Theory Tech., vol. MTT-25, p. $367,1977$.

[40] I. Ury, K. Y. Lau, N. Bar-Chaim, and A. Yariv, "Very high frequency GaAlAs laser-FET monolithic integrated circuit," Appl. Phys. Lett., Aug. 1982.

[41] S. D. Personick, N. L. Rhodes, D. C. Hanson, and K. H. Chan, "Contrasting fiber optic component design requirements in telecommunication analog and local data communicating applications," Proc. IEEE, vol. 68, pp. 1254-1262, 1980.

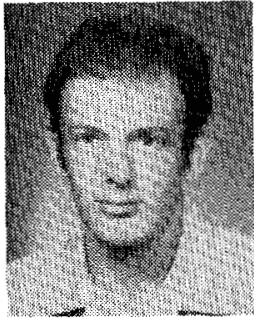

Uziel Koren was born in Jerusalem, Israel, in 1946. He received the B.Sc. degree in physics and mathematics and the M.Sc. degree in physics from the Hebrew University, Jerusalem, Israel, and the Ph.D. degree from the Weizmann Institute' of Science, Rehovot, Israel, in 1978.

He was a Research Fellow at the Weizmann Institute of Science until 1980, where he was engaged in research on infrared detectors and systems. Currently he is a Research Associate at the California Institute of Technology, Pasadena, where he is working on semiconductor optoelectronic devices.



Shlomo Margalit was born in Haifa, Israel, on July 29,1941 . He received the B.Sc., M.Sc., and D.Sc degrees from the Technion-Israel Institute of Technology, Haifa, Israel, in 1965, 1968 , and 1971, respectively.

Since 1971 he has been a Faculty Member of the Department of Electrical Engineering, Technion-Israel Institute of Technology. In 1980 he became an Associate Professor. During the academic years 1977-1978 and 1981-1982 he was a Visiting Associate at the California Institute of Technology, Pasadena. His research interests are in the field of semiconductor devices.

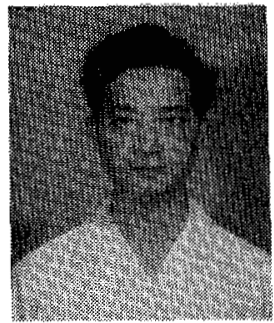

T. R. Chen was born in January 1940. He graduated from Wuhan University, Wuhan, China, in 1962.

He joined the faculty of Chengdu Institute of Radio Engineering, where he conducted research in gas lasers, optical communication, and cavity theory. Since 1980 he has been a Visiting Associate at the California Institute of Technology. His interests include semiconductor lasers, optoelectronic integration, and optical communication.

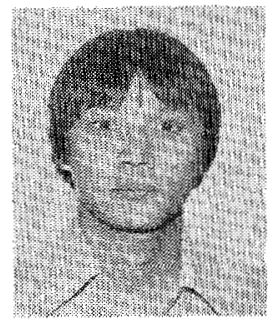

K. L. Yu received the B.S. degree in physics and the M.S. degree in applied physics from the California Institute of Technology, Pasadena in 1979 .

Currently he is working on a doctoral dissertation in the field of optoelectronic devices for optical communication at the California Institute of Technology.

$\mathrm{He}$ is a member of the American Physical Society and Tau Beta $\mathrm{Pi}$. 
Amnon Yariv (S'56-M'59-F'70), for a photograph and biography, see p. 337 of the March 1982 issue of this JoURNAL.

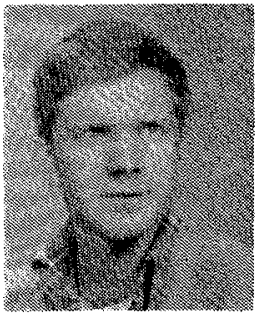

Nadav Bar-Chaim was born in Nathanya, Istael in 1946. He received the B.Sc. degree in mathematics and physics from the Hebrew University, Jerusalem, Israel, in 1967, and the M.S. degree in physics and the Ph.D. degree in electrical engineering from Tel-Aviv University, TelAviv, Israel, in 1973 and 1978, respectively.

In 1978 he joined the California Institute of Technology, Pasadena, as a Postdoctoral Fellow, where his work included research in semiconductor lasers and integrated optoelectronic circuits. In 1981 he joined Ortel Corporation, Alhambra, CA.
Kam Y. Lau (M'78), for a photograph and biography, see p. 1361 of the September 1982 issue of this JourNaL.



Israel Ury was born in St. Louis, MO, in 1956. He received the B.Sc. and M.S. degrees in electrical engineering from the University of California, Los Angeles, and the Ph.D. degree in applied physics from the California Institute of Technology, Pasadena, in 1975, 1976, and 1980 , respectively.

While at the California Institute of Technology he was involved with research in integrated optoelectronics. Currently, he is President of Ortel Corporation, Alhambra, CA.

\title{
Optical Phase Modulation in an Injection Locked AlGaAs Semiconductor Laser
}

\author{
SOICHI KOBAYASHI, MEMBER, IEEE, AND TATSUYA KIMURA, SENIOR MEMBER, IEEE
}

\begin{abstract}
Optical phase modulation obtained by injecting coherent $\mathrm{CW}$ light into a directly frequency-modulated semiconductor laser is reported. Phase modulation at up to a $1 \mathrm{GHz}$ modulation frequency has been obtained without compression for a $1.4 \mathrm{GHz}$ half locking bandwidth. Phase deviation can be represented by the ratio of the original FM deviation to the locking half bandwidth. The phase deviation normalized by the frequency deviation is inversely proportional to the cutoff modulation frequency. A static phase shift of $\pi$ took place with a $0.48 \mathrm{~mA}$ drive current change in the injection locked laser.

Reduction in FM noise by means of CW light injection and FM noise accumulation in cascaded injection locked laser amplifiers are discussed, together with the optimum design for an injection locked repeater system.
\end{abstract}

\section{INTRODUCtion}

$\mathrm{C}$ OHERENT optical transmission systems are attractive as future systems with wide repeater spacing and large information capacity [1]. The amplitude, frequency, or phase of the coherent optical carrier is modulated and then demodulated through a homodyne or heterodyne scheme. The possibility of a coherent FSK signal transmission system using a semiconductor laser transmitter and an independent local oscillator for heterodyning has successfully been demonstrated [2]. A coherent PSK signal transmission system is desired as a more advanced technology because of the low receiving power level in the PSK-homodyne or -heterodyne detection scheme [1].

Manuscript received February 17, 1982

The authors are with the Musashino Electrical Communication Laboratory, Nippon Telegraph and Telephone Public Corporation, Musashinoshi, Tokyo, Japan.
Injection locking in semiconductor lasers has been studied [3], [4] for coherent optical system applications. The bandwidthversus-gain relation of injection locked semiconductor laser amplifiers, amplification of FM optical signals, and single mode operations in directly modulated semiconductor lasers injected with CW light, have been reported [5]-[10].

Direct optical frequency modulation in semiconductor lasers has been demonstrated and the broad-band modulation frequency characteristics of different types of laser structures have been studied [11], [12].

The present paper reports experimental studies on optical phase modulation (PM) obtained by injecting $\mathrm{CW}$ coherent light into a directly frequency-modulated (FM) AlGaAs double heterostructure semiconductor laser. Measurement systems and procedures for the induced PM will be described in Section II. In Section III, the results obtained in the static phase shift and dynamic PM modulation by injection locking will be shown. Analytical treatment will be described in Section IV. Reduction in semiconductor laser FM noise by injection locking will be discussed in Section $\mathrm{V}$.

\section{Measurement Systems and Procedures}

\section{A. Experimental Setup}

An experimental setup to study phase modulation is shown in Fig. 1. Two double heterostructure AlGaAs semiconductor lasers $(L D 1, L D 2)$, with an identical cavity length, operate continuously in a single longitudinal mode at $840 \mathrm{~nm}$. These lasers have channeled substrate planar (CSP) geometry [13]. Their oscillation thresholds are $I_{\mathrm{th}, 1}=62 \mathrm{~mA}$ and $I_{\mathrm{th}, 2}=6 \mathrm{~mA}$. They are mounted individually on copper heat sinks whose 